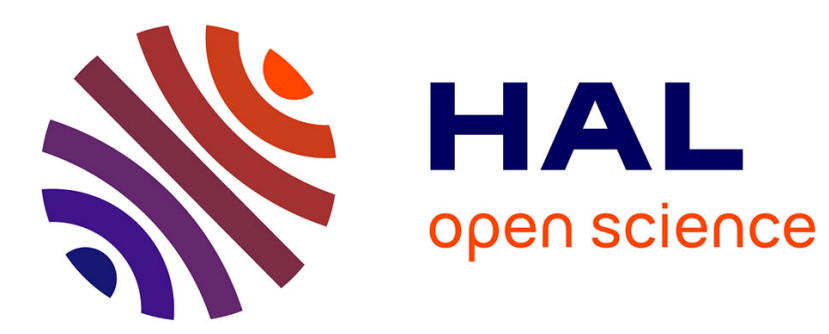

\title{
Switching controller for stabilization of linear systems with switched time-varying delays
}

Wenjuan Jiang, Emilia Fridman, Alexandre Kruszewski, Jean-Pierre Richard

\section{To cite this version:}

Wenjuan Jiang, Emilia Fridman, Alexandre Kruszewski, Jean-Pierre Richard. Switching controller for stabilization of linear systems with switched time-varying delays. CDC'09, 48th IEEE Conf. on Decision and Control, Dec 2009, Shanghai, China. inria-00419360

\section{HAL Id: inria-00419360 https://hal.inria.fr/inria-00419360}

Submitted on 23 Dec 2009

HAL is a multi-disciplinary open access archive for the deposit and dissemination of scientific research documents, whether they are published or not. The documents may come from teaching and research institutions in France or abroad, or from public or private research centers.
L'archive ouverte pluridisciplinaire HAL, est destinée au dépôt et à la diffusion de documents scientifiques de niveau recherche, publiés ou non, émanant des établissements d'enseignement et de recherche français ou étrangers, des laboratoires publics ou privés. 


\title{
Switching controller for stabilization of linear systems with switched time-varying delays
}

\author{
Wenjuan Jiang, Emilia Fridman, Alexandre Kruszewski and Jean-Pierre Richard
}

\begin{abstract}
This paper considers interval time-varying delay systems with delayed estimation of the delay. This case is often encountered in the Networked Control Systems (NCS) field. Based on Lyapunov-Krasovskii functional methods and linear matrix inequality (LMI) techniques, a switching state feedback controller is designed to guarantee the exponential stability. The controller switches according to the measured time-delay which is itself delayed. The global stability of the switching closed loop is guaranteed if some dwell time conditions are satisfied.

Keywords: time-varying delay, switching systems, delayed switching signal, Lyapunov-Krasovskii functional, LMI
\end{abstract}

\section{INTRODUCTION}

A variety of stability and control techniques have been developed for general time delay systems [2], [14], [16]. Of course, when a delay is introduced in a control loop, the achievable performance (speed, robustness) highly depends on the delay value. In the case of a time-varying delay, the performance one can guaranty generally depends on the interval of variation of the delay. To give an example, controlling a system through a communication network induces variable time delays and the resulting performance depends on the quality of service (QoS) the network can offer. Designing a robust controller on the basis of the worst case (highest delay, lowest QoS) is possible, but such a cautious solution may yield poor speed performance if the delay is not as big as expected. Then, it is worth designing a switching controller that adapts to different intervals of delay variation. However, in this case, the usual techniques for time-delay systems cannot be directly applied and one has to go to switched systems techniques.

Switched systems are dynamical hybrid systems consisting of a family of continuous-time subsystems and a logical rule that orchestrates the switching between them [9], [11]. In general, the stability of the subsystems themselves are not sufficient for the stability of the overall system. A lot of studies have dealt with the stability analysis and design of switched systems [1],

W.-J. Jiang, A. Kruszewski and J.-P. Richard are with LAGIS CNRS UMR 8146, Ecole Centrale de Lille, BP 48, 59651 Villeneuve d Ascq Cedex, France. J.-P. Richard is also with Equipe-Projet ALIEN, INRIA.

E. Fridman is with School of Electrical Engineering, Tel Aviv University, Tel Aviv 69978, Israel.

E-mail: \{wenjuan.jiang, Alexandre.Kruszewski, jeanpierre.richard \}@ec-lille.fr, emilia@eng.tau.ac.il
[7], [12], [19]. According to Liberzon and Morse, these problems can be divided into three basic problems: (1) Construction of a common Lyapunov functional to guarantee the stability for arbitrary switching sequences [10], [13], [18]; (2) Multiple Lyapunov functions are applied to the stability for certain useful classes of switching sequences; (3) Construction of stabilizing switching sequences.

Most of the results of switching systems are based on the assumption that the detection of the switching signal is instant, while, in many real systems, the signal can only be detected after a time period. For example, the size of time-delay in the case of Internet can be measured only after a RTT (Round-trip time) of packet exchange. In [19], the delayed signal is considered but without time-delay in the system. The uncertain switching signal is considered for discrete switching systems in [8], which is easier to be resolved than the case of continuous systems.

Single [10], [17] and multiple Lyapunov-Krasovskii Functionals (LKFs) analysis methods are the most frequently applied in the stabilization of the whole system. A common Lyapunov-Krasovskii Functional can ensure the stability with arbitrary switching but it does not always exist. As an alternative, dwell-time based switching is considered in [7] but time delay is not considered, while in [1], [20], the time delay is constant one.

In this paper, a linear switched time-delay system with delayed switching signals is considered, where the uncertain time-varying delay switches between the known two sequential intervals. A state feedback switching controller is designed to guarantee the exponential stability of the switching modes. Lyapunov-Krasovskii Functionals (LKFs) analysis methods are applied in the stabilization analysis. We derive delay-dependent stability conditions in a convex way, following the idea of [15].

Notation: Throughout the paper the superscript ' $T$ ', stands for matrix transposition, $\mathscr{R}^{n}$ denotes the $n$ dimensional Euclidean space with vector norm $\|\cdot\|, \mathscr{R}^{n \times m}$ is the set of all $n \times m$ real matrices, and the notation $P>0$, for $P \in \mathscr{R}^{n \times n}$ means that $P$ is symmetric and positive definite. The symmetric elements of the symmetric matrix will be denoted by $* . L_{2}$ is the space of square integrable functions $v:[0, \infty) \rightarrow R^{n}$ with the norm 
$\|v\|_{L_{2}}=\left[\int_{0}^{\infty}\|v(t)\|^{2} d t\right]^{1 / 2}$.

\section{PROBLEM FORMULATION AND PRELIMINARIES}

Consider a state feedback controller to stabilize the following linear time-delay system:

$$
\dot{x}(t)=A x(t)+B u(t-\tau(t))
$$

with $\tau(t)$, a fast varying interval delay with the bounds as $\left[h_{\min }, h_{\max }\right]$. We know that the stabilization is generally easier (we mean here, to guarantee a higher exponential rate) if the delay is small. So the interval can be divided into several smaller ones in correspondence with the switched controller. To simplify the problem, here two switching modes are considered, but it can be easily extended to more modes.

The switching controller to be considered is:

$$
u(t)=\left\{\begin{array}{l}
K_{1} x(t), \tau(t) \in\left[h_{1}, h_{2}\right] \\
K_{2} x(t), \tau(t) \in\left[h_{2}, h_{3}\right]
\end{array}\right.
$$

So, the closed-loop system can be written as follows:

$$
\begin{aligned}
\dot{x}(t)= & A x(t)+\chi_{\left[h_{1}, h_{2}\right]}(\tau) B K_{1} x(t-\tau(t)) \\
& +\left(1-\chi_{\left[h_{1}, h_{2}\right]}\right)(\tau) B K_{2} x(t-\tau(t)),
\end{aligned}
$$

where $\tau \in\left[h_{1}, h_{2}\right] \cup\left[h_{2}, h_{3}\right]$ and where $\chi_{\left[h_{1}, h_{2}\right]}: R \rightarrow$ $\{0,1\}$ is the characteristic function of $\left[h_{1}, h_{2}\right]$

$$
\chi_{\left[h_{1}, h_{2}\right]}(s)= \begin{cases}1, & \text { if } s \in\left[h_{1}, h_{2}\right] \\ 0, & \text { otherwise. }\end{cases}
$$

\section{EXPONENTIAL STABILITY OF THE SWITCHED SYSTEM: ARBITRARY SWITCHING}

Consider the switched system (2) and make $A_{i}=$ $B K_{i}, i=1,2$. In the following, the notation $x_{t}$ stands for $x(t)$. Applying the following LKF:

$$
\begin{aligned}
& V\left(t, x_{t}, \dot{x}_{t}\right)=x^{T}(t) P x(t) \\
& +\sum_{i=0}^{2} \int_{t-h_{i+1}}^{t-h_{i}} e^{2 \alpha(s-t)} x^{T}(s) S_{i} x(s) d s \\
& +\sum_{i=0}^{2}\left(h_{i+1}-h_{i}\right) \int_{-h_{i+1}}^{-h_{i}} \int_{t+\theta}^{t} e^{2 \alpha(s-t)} \dot{x}^{T}(s) R_{i} \dot{x}(s) d s d \theta
\end{aligned}
$$

where $h_{0}=0, P>0$ and $R_{i}, S_{i} \geq 0$. Saying that the system (2) is exponentially stable means that there exist a positive function $W(t, x(t), \dot{x}(t))$ such that:

$$
W\left(t, x_{t}, \dot{x}_{t}\right) \leq e^{-2 \alpha\left(t-t_{0}\right)} W\left(t, x_{t_{0}}, \dot{x}_{t_{0}}\right)
$$

It can be ensured by using the LKFs (3) and by checking if:

$$
\dot{V}\left(t, x_{t}, \dot{x}_{t}\right)+2 \alpha V\left(t, x_{t}, \dot{x}_{t}\right)<0
$$

After the differentiation of the function (3), the system is exponentially stable if:

$$
\begin{aligned}
& \dot{V}\left(t, x_{t}, \dot{x}_{t}\right)+2 \alpha V\left(t, x_{t}, \dot{x}_{t}\right) \leq 2 x^{T}(t) P \dot{x}(t) \\
& +2 \alpha x^{T}(t) P x(t)+\dot{x}^{T}(t)\left[\sum_{i=0}^{2}\left(h_{i+1}-h_{i}\right)^{2} R_{i}\right] \dot{x}(t) \\
& -\sum_{i=0}^{2}\left(h_{i+1}-h_{i}\right) e^{-2 \alpha h_{i+1} \int_{t-h_{i+1}}^{t-h_{i}} \dot{x}^{T}(s) R_{i} \dot{x}(s) d s} \\
& +\sum_{i=0}^{2} x\left(t-h_{i}\right)^{T} e^{-2 \alpha h_{i}} S_{i} x\left(t-h_{i}\right) \\
& -\sum_{i=0}^{2} e^{-2 \alpha h_{i+1}} x^{T}\left(t-h_{i+1}\right) S_{i} x\left(t-h_{i+1}\right) .
\end{aligned}
$$

We start with the case of $\chi=1$, i.e. of $\tau \in\left[h_{1}, h_{2}\right]$. Noticing that:

$$
\begin{aligned}
\int_{t-h_{i+1}}^{t-h_{i}} \dot{x}^{T}(s) R_{i} \dot{x}(s) d s & =\int_{t-h_{i+1}}^{t-\tau(t)} \dot{x}^{T}(s) R_{i} \dot{x}(s) d s \\
& +\int_{t-\tau(t)}^{t-h_{i}} \dot{x}^{T}(s) R_{i} \dot{x}(s) d s
\end{aligned}
$$

where $i=0,2$ and applying the Jensen's inequality [4]

$$
\begin{aligned}
& \int_{t-h_{i+1}}^{t-h_{i}} \dot{x}^{T}(s)\left[\left(h_{i+1}-h_{i}\right) R_{i}\right] \dot{x}(s) d s \\
& \geq \int_{t-h_{i}}^{t-h_{i+1}} \dot{x}^{T}(s) d s R_{i} \int_{t-h_{i+1}}^{t-h_{i}} \dot{x}(s) d s, \\
& \int_{t-\tau(t)}^{t-h_{j}} \dot{x}^{T}(s)\left(h_{j+1}-h_{j}\right) R_{j} \dot{x}(s) d s \\
& \geq \frac{h_{j+1}-h_{j}}{\tau-h_{j}} \int_{t-\tau(t)}^{t-h_{j}} \dot{x}^{T}(s) d s R_{j} \int_{t-\tau(t)}^{t-h_{j}} \dot{x}(s) d s, \\
& \int_{t-h_{j+1}}^{t-\tau(t)} \dot{x}^{T}(s)\left[\left(h_{j+1}-h_{j}\right) R_{j}\right] \dot{x}(s) d s \\
& \geq \frac{h_{j+1}-h_{j}}{h_{j+1}-\tau} \int_{t-h_{j+1}}^{t-\tau(t)} \dot{x}^{T}(s) d s R_{j} \int_{t-h_{j+1}}^{t-\tau(t)} \dot{x}(s) d s,
\end{aligned}
$$

where $j=1, i=0,2$. Here for $\tau \rightarrow h_{j}$ we understand by

$$
\begin{aligned}
\frac{1}{\tau(t)-h_{j}} \int_{t-\tau(t)}^{t-h_{j}} \dot{x}(s) d s & =\lim _{\tau \rightarrow h_{j}} \frac{1}{\tau(t)-h_{j}} \int_{t-\tau(t)}^{t-h_{j}} \dot{x}(s) d s \\
& =\dot{x}\left(t-h_{j}\right) .
\end{aligned}
$$

For $h_{j+1}-\tau(t) \rightarrow 0$ the vector $\frac{1}{h_{j+1}-\tau(t)} \int_{t-h_{j+1}}^{t-\tau(t)} \dot{x}(s) d s$ is defined similarly as $\dot{x}\left(t-h_{j+1}\right)$.

Denoting

$$
\begin{aligned}
v_{j 1} & =\frac{1}{\tau-h_{j}} \int_{t-\tau(t)}^{t-h_{j}} \dot{x}(s) d s \\
v_{j 2} & =\frac{1}{h_{j+1}-\tau} \int_{t-h_{j+1}}^{t-\tau(t)} \dot{x}(s) d s
\end{aligned}
$$

we obtain

$$
\begin{aligned}
& \dot{V}\left(t, x_{t}, \dot{x}_{t}\right)+2 \alpha V\left(t, x_{t}, \dot{x}_{t}\right) \leq 2 x^{T}(t) P \dot{x}(t) \\
& \left.+2 \alpha x^{T}(t) P x(t)+\dot{x}^{T}(t) \sum_{i=0}^{2}\left(h_{i+1}-h_{i}\right)^{2} R_{i}\right) \dot{x}(t) \\
& +\sum_{i=0}^{2} e^{-2 \alpha h_{i}} x^{T}\left(t-h_{i}\right) S_{i} x\left(t-h_{i}\right) \\
& -\sum_{i=0}^{2} e^{-2 \alpha h_{i+1}} x^{T}\left(t-h_{i+1}\right) S_{i} x\left(t-h_{i+1}\right) \\
& -\left[x(t)-x\left(t-h_{1}\right)\right]^{T} e^{-2 \alpha h_{1}} R_{0}\left[x(t)-x\left(t-h_{1}\right)\right] \\
& -\left[x\left(t-h_{2}\right)-x\left(t-h_{3}\right)\right]^{T} e^{-2 \alpha_{1} h_{3}} R_{2}\left[x\left(t-h_{2}\right)-x\left(t-h_{3}\right)\right] \\
& -\left(\tau-h_{j}\right)\left(h_{j+1}-h_{j}\right) v_{j 1}^{T} e^{-2 \alpha h_{2}} R_{j} v_{j 1} \\
& -\left(h_{j+1}-\tau\right)\left(h_{j+1}-h_{j}\right) v_{j 2}^{T} e^{-2 \alpha h_{2}} R_{j} v_{j 2} .
\end{aligned}
$$

We note that in the latter bound we can substitute for $\dot{\tau}$ its upper bound $d_{2}$.

We insert free-weighting $n \times n$-matrices [5], [6] by adding the following expressions to $\dot{V}$ :

$$
\begin{aligned}
0 & =2\left[x^{T}(t) Y_{1 j}^{T}+\dot{x}^{T}(t) Y_{2 j}^{T}+x^{T}(t-\tau) T_{j}^{T}\right]\left[-x\left(t-h_{j}\right)\right. \\
& \left.+x(t-\tau)+\left(\tau-h_{j}\right) v_{j 1}\right] \\
0 & =2\left[x^{T}(t) Z_{1 j}^{T}+\dot{x}^{T}(t) Z_{2 j}^{T}\right]\left[x\left(t-h_{j+1}\right)\right. \\
& \left.+\left(h_{j+1}-\tau\right) v_{j 2}-x(t-\tau)\right] .
\end{aligned}
$$

We use further the descriptor method [3], where the right-hand side of the expression

$$
0=2\left[x^{T}(t) P_{2 j}^{T}+\dot{x}^{T}(t) P_{3 j}^{T}\right]\left[A x(t)+A_{1} x(t-\tau(t))-\dot{x}(t)\right],
$$


with some $n \times n$-matrices $P_{2 j}, P_{3 j}$ is added into the righthand side of (9).

Setting $\quad \eta_{j}(t)=\operatorname{col}\left\{x(t), \dot{x}(t), x\left(t-h_{1}\right), x(t-\right.$ $\left.\left.h_{2}\right), v_{j 1}, v_{j 2}, x(t-\tau), x\left(t-h_{3}\right)\right\}$, where $j=1$, we obtain that along the switched system (2)

$$
\dot{V}\left(t, x_{t}, \dot{x}_{t}\right)+2 \alpha V\left(t, x_{t}, \dot{x}_{t}\right) \leq \eta_{j}^{T}(t) \Phi \eta_{j}(t)<0,
$$

if the LMI (13) is feasible, where $\dot{\tau}=d_{2}$ and

$$
\begin{aligned}
& \tilde{R}_{i}=e^{-2 \alpha h_{i+1}} R_{i}, \tilde{S}_{i}=e^{-2 \alpha h_{i+1}} S_{i}, \hat{S}_{i}=e^{-2 \alpha h_{i}} S_{i}, \\
& \Phi_{11}^{j}=A^{T} P_{2 j}+P_{2 j}^{T} A+S_{0}-e^{-2 \alpha h_{1}} R_{0}+2 \alpha P, \\
& \Phi_{12}^{j}=P-P_{2 j}^{T}+A^{T} P_{3 j}, \\
& \Phi_{22}^{j}=-P_{3 j}-P_{3 j}^{T}+\sum_{i=0}^{2}\left(h_{i+1}-h_{i}\right)^{2} R_{i}, \\
& \Phi_{16}^{j}=P_{2 j}^{T} A_{j}, \Phi_{26}^{j}=P_{3 j}^{T} A_{j}, \phi_{3}^{(1)}=-\left(\tilde{S}_{0}+\tilde{R}_{0}-\hat{S}_{1}\right), \\
& \phi_{4}^{(1)}=-\left(\tilde{S}_{1}+\tilde{R}_{2}-\hat{S}_{2}\right), \\
& \phi_{5}^{(1)}=-\left(h_{2}-h_{1}\right)\left(\tau-h_{1}\right) \tilde{R}_{1}, \\
& \phi_{6}^{(1)}=-\left(h_{2}-h_{1}\right)\left(h_{2}-\tau\right) \tilde{R}_{1} .
\end{aligned}
$$

The latter condition leads for $\tau \rightarrow h_{1}$ and for $\tau \rightarrow$ $h_{2}$ to the following LMIs (14) and (15), where we deleted the zero column and the zero row. Denote by $\eta_{1 i}(t)=\operatorname{col}\left\{x(t), \dot{x}(t), x\left(t-h_{1}\right), x\left(t-h_{2}\right), v_{j i}, x(t-\right.$ $\left.\tau), x\left(t-h_{3}\right)\right\}, i=1,2$.

Then (14), (15) imply (12) because

$$
\begin{aligned}
& \frac{h_{2}-\tau}{h_{2}-h_{1}} \eta_{12}^{T}(t) \Psi_{1} \eta_{12}(t)+\frac{\tau-h_{1}}{h_{2}-h_{1}} \eta_{11}^{T}(t) \Psi_{2} \eta_{11}(t) \\
& =\eta_{1}^{T}(t) \Psi_{\mid \chi=1} \eta_{1}(t)<0 .
\end{aligned}
$$

Thus, $\Psi_{\mid \chi=1}$ is convex in $\tau \in\left[h_{1}, h_{2}\right]$.

For $\chi=0$, i.e. for $\tau \in\left[h_{2}, h_{3}\right]$ applying the same arguments and representations with $j=2$ and $i=0,1$, we arrive to the following:

$$
\left.\dot{V}\left(t, x_{t}, \dot{x}_{t}\right)\right|_{\chi=0} \leq\left.\eta_{2}^{T}(t) \Psi\right|_{\chi=0} \eta_{2}(t),
$$

with the LMI (17) and where

$$
\begin{aligned}
& \phi_{3}^{(2)}=-\left(\tilde{S}_{0}+\tilde{R}_{0}+\tilde{R}_{1}-\hat{S}_{1}\right), \\
& \phi_{4}^{(2)}=-\left(\tilde{S}_{1}+\tilde{R}_{1}-\hat{S}_{2}\right), \\
& \phi_{5}^{(2)}=-\left(h_{3}-h_{2}\right)\left(\tau-h_{2}\right) \tilde{R}_{2}, \\
& \phi_{6}^{(2)}=-\left(h_{3}-h_{2}\right)\left(h_{3}-\tau\right) \tilde{R}_{2} .
\end{aligned}
$$

We note that $\left.\Psi\right|_{\chi=0}$ is convex in $\tau \in\left[h_{2}, h_{3}\right]$ and, thus, for the feasibility of LMI (17) (given in next page) it is sufficient to verify this LMI (19) and (20) for $\tau \rightarrow h_{2}$ and for $\tau \rightarrow h_{3}$ : Then, along the switched system (2)

$$
\begin{aligned}
& \dot{V}\left(t, x_{t}, \dot{x}_{t}\right) \leq\left.\chi_{\left[h_{1}, h_{2}\right]}(\tau) \eta_{1}^{T}(t) \Psi\right|_{\chi=1} \eta_{1}(t) \\
& +\left.\left(1-\chi_{\left[h_{1}, h_{2}\right]}(\tau)\right) \eta_{2}^{T}(t) \Psi\right|_{\chi=0} \eta_{2}(t)<0,
\end{aligned}
$$

if the four LMIs (14), (15), (19) and (20) are feasible, thus, the following result is obtained.

Theorem 1: Let there exist $n \times n$-matrices $P>0$, $R_{i}>0, S_{i}>0, i=0,1,2, P_{2 j}, P_{3 j}, Y_{1 j}, Y_{2 j}, T_{j}, Z_{1 j}$ and $Z_{2 j}, j=1,2$ such that the four LMIs (14), (15), (19) and (20) with notations given in (16),(18) are feasible. Then the switched system (2) is asymptotically stable for all delays $\tau_{i} \in\left[h_{i}, h_{i+1}\right], i=1,2$ with a decay rate $\alpha$.

\section{QOS ADAPTED SWITCHING GAIN STRATEGY}

As in the section III, in order to find the common LKFs, the same $\alpha$ has to be chosen for the two modes, that is to say, the performance cannot be better in the case of small time-delay. Some other works, like [10], [18], separately search different $\alpha$ for each mode but at the same time search the uniform stability for both of the zones. This method is conservative and the common LKFs cannot be definitely found. In this paper, different LKFs are applied with a minimum dwell time ensuring the stability.

Not using a common LKF reduces the conservatism as each mode can have a completely different behavior. This reduction allows, in most cases, getting better performances or robustness.

The stability analysis is performed by using different LKFs for different mode. In order to make the LKFs comparable, their structure are chosen as follows:

$$
\begin{aligned}
& V_{j}\left(t, x_{t}, \dot{x}_{t}\right)=x^{T}(t) P_{j} x(t) \\
& +\sum_{i=0}^{2} \int_{t-h_{i+1}}^{t-h_{i}} e^{2 \alpha_{j}(s-t)} x^{T}(s) S_{i j} x(s) d s \\
& +\sum_{i=0}^{2}\left(h_{i+1}-h_{i}\right) \int_{-h_{i+1}}^{-h_{i}} \int_{t+\theta}^{t} e^{2 \alpha_{j}(s-t)} \dot{x}^{T}(s) R_{i j} \dot{x}(s) d s d \theta
\end{aligned}
$$

where $j$ represents the mode, $V_{1}$ for $\tau_{1} \in\left[h_{1}, h_{2}\right]$ and $V_{2}$ for $\tau_{2} \in\left[h_{2}, h_{3}\right]$.

These LKFs consider the delay over the two zones. Without lack of generality, it can be used to analyze the closed loop when the delay belongs to a specific zone i.e. when the controller is fixed in one mode. Note that if $R_{2}=0, S_{2}=0$ then the previous LKF (3) is recovered for a time-delay in $\tau(t) \in\left[h_{1}, h_{2}\right]$.

In the following parts, delayed switching signals are considered. The minimum dwell time for each mode is calculate to guarantee the global system stability.

\section{A. QoS adapted performance}

As showed in Fig. 1, the system is supposed to switch at the moment of $t_{1}$ and $t_{3}$, but the switching signals can only be detected by the system after the period of time $\left(t_{2}-t_{1}\right)$ and $\left(t_{4}-t_{3}\right)$. Even if the system is stable in the two modes, the delayed signals can make the system unstable (wrong control gain). In order to make the global system stable, the values of the LKFs should be in the decreasing sequence as showed in Fig. 1. The two modes switching system with delayed switching signals can be modeled as four modes, which is showed in Fig. 2. Here the two principle modes $S S_{1}$ and $S S_{2}$ are considered as exponential stable and the stability of the two modes $S U_{1}$ and $S U_{2}$ cannot be proved because of the wrong gains. 


$$
\begin{aligned}
& \left.\Psi\right|_{\chi=1}=\left[\begin{array}{cccccccc}
\Phi_{11}^{1}-(1-\tau) X & \Phi_{12}^{1} & \tilde{R}_{0}-Y_{11}^{T} & Z_{11}^{T} & \left(\tau-h_{1}\right) Y_{11}^{T} & \left(h_{2}-\tau\right) Z_{11}^{T} & Y_{11}^{T}-Z_{11}^{T}+\Phi_{16}^{j} & 0 \\
* & \Phi_{22}^{1} & -Y_{21}^{T} & Z_{21}^{T} & \left(\tau-h_{1}\right) Y_{21}^{T} & \left(h_{2}-\tau\right) Z_{21}^{T} & Y_{21}^{T}-Z_{21}^{T}+\Phi_{26}^{j} & 0 \\
* & * & \phi_{3}^{(1)} & 0 & 0 & 0 & -T_{1} & 0 \\
* & * & * & \phi_{4}^{(1)} & 0 & 0 & 0 & \tilde{R}_{2} \\
* & * & * & * & \phi_{5}^{(1)} & 0 & \left(\tau-h_{1}\right) T_{1} & 0 \\
* & * & * & * & * & \phi_{6}^{(1)} & 0 & 0 \\
* & * & * & * & * & * & T_{1}+T_{1}^{T} & 0 \\
* & * & * & * & * & * & * & \left.* \tilde{S}_{2}+\tilde{R}_{2}\right)
\end{array}\right]<0, \\
& \Psi_{11}=\left[\begin{array}{ccccccc}
\Phi_{11}^{1} & \Phi_{12}^{1} & \tilde{R}_{0}-Y_{11}^{T} & Z_{11}^{T} & \left(h_{2}-h_{1}\right) Z_{11}^{T} & Y_{11}^{T}-Z_{11}^{T}+\Phi_{16}^{j} & 0 \\
* & \Phi_{22 \mid \tau=h_{1}}^{1} & -Y_{21}^{T} & Z_{21}^{T} & \left(h_{2}-h_{1}\right) Z_{21}^{T} & Y_{21}^{T}-Z_{21}^{T}+\Phi_{26}^{j} & 0 \\
* & * & \phi_{3}^{(1)} & 0 & 0 & -T_{1} & 0 \\
* & * & * & \phi_{4}^{(1)} & 0 & 0 & \tilde{R}_{2} \\
* & * & * & * & \phi_{6}^{(1)}{ }_{\mid \tau=h} & 0 & 0 \\
* & * & * & * & * & T_{1}+T_{1}^{T} & 0 \\
* & * & * & * & * & * & -\left(\tilde{S}_{2}+\tilde{R}_{2}\right)
\end{array}\right]<0,
\end{aligned}
$$

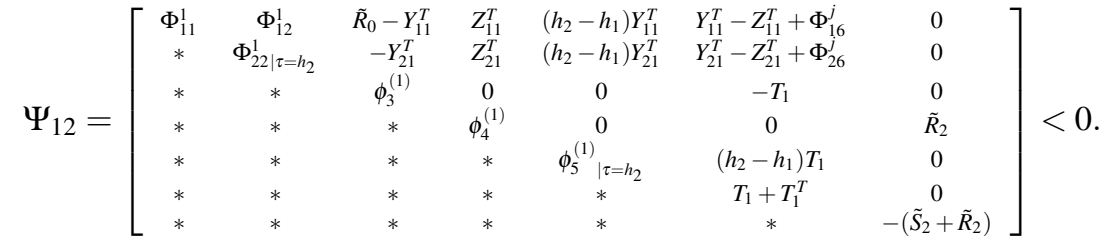

$$
\begin{aligned}
& \left.\Psi\right|_{\chi=0}=\left[\begin{array}{cccccccc}
\Phi_{11}^{2} & \Phi_{12}^{2} & \tilde{R}_{0} & -Y_{12}^{T} & \left(\tau-h_{2}\right) Y_{12}^{T} & \left(h_{3}-\tau\right) Z_{12}^{T} & Y_{12}^{T}-Z_{12}^{T}+\Phi_{16}^{j} & Z_{12}^{T} \\
* & \Phi_{22}^{2} & 0 & -Y_{22}^{T} & \left(\tau-h_{2}\right) Y_{22}^{T} & \left(h_{3}-\tau\right) Z_{22}^{T} & Y_{22}^{T}-Z_{22}^{T}+\Phi_{26}^{j} & Z_{22}^{T} \\
* & * & \phi_{3}^{(2)} & \tilde{R}_{1} & 0 & 0 & 0 & 0 \\
* & * & * & \phi_{4}^{(2)} & 0 & 0 & -T_{2} & 0 \\
* & * & * & * & \phi_{5}^{(2)} & 0 & \left(\tau-h_{2}\right) T_{2} & 0 \\
* & * & * & * & * & \phi_{6}^{(2)} & 0 & 0 \\
* & * & * & * & * & * & -(1-\tau) Q+T_{2}+T_{2}^{T} & 0 \\
* & * & * & * & * & * & * & -\tilde{S}_{2}
\end{array}\right]<0, \\
& \Psi_{01}=\left[\begin{array}{ccccccc}
\Phi_{11}^{2} & \Phi_{12}^{2} & \tilde{R}_{0} & -Y_{12}^{T} & \left(h_{3}-h_{2}\right) Z_{12}^{T} & Y_{12}^{T}-Z_{12}^{T}+\Phi_{16}^{j} & Z_{12}^{T} \\
* & \Phi_{22 \mid \tau=h}^{2} & 0 & -Y_{22}^{T} & \left(h_{3}-h_{2}\right) Z_{22}^{T} & Y_{22}^{T}-Z_{22}^{T}+\Phi_{26}^{j} & Z_{22}^{T} \\
* & * & \phi_{3}^{(2)} & \tilde{R}_{1} & 0 & 0 & 0 \\
* & * & * & \phi_{4}^{(2)} & 0 & -T_{2} & 0 \\
* & * & * & * & \phi_{6}^{(2)} \mid \tau=h_{2} & 0 & 0 \\
* & * & * & * & * & T_{2}+T_{2}^{T} & 0 \\
* & * & * & * & * & * & -\tilde{S}_{2}
\end{array}\right]<0, \\
& \Psi_{02}=\left[\begin{array}{ccccccc}
\Phi_{11}^{2} & \Phi_{12}^{2} & \tilde{R}_{0} & -Y_{12}^{T} & \left(h_{3}-h_{2}\right) Y_{12}^{T} & Y_{12}^{T}-Z_{12}^{T}+\Phi_{16}^{j} & Z_{12}^{T} \\
* & \Phi_{22}^{2} \mid \tau=h_{3} & 0 & -Y_{22}^{T} & \left(h_{3}-h_{2}\right) Y_{22}^{T} & Y_{22}^{T}-Z_{22}^{T}+\Phi_{26}^{j} & Z_{22}^{T} \\
* & * & \phi_{3}^{(2)} & \tilde{R}_{1} & 0 & 0 & 0 \\
* & * & * & \phi_{4}^{(2)} & 0 & -T_{2} & 0 \\
* & * & * & * & \phi_{5}^{(2)}{ }_{\mid \tau=h} & \left(h_{3}-h_{2}\right) T_{2} & 0 \\
* & * & * & * & * & T_{2}+T_{2}^{T} & 0 \\
* & * & * & * & * & * & -\tilde{S}_{2}
\end{array}\right]<0 .
\end{aligned}
$$

The four switch modes can be modeled as follows:

$$
\begin{aligned}
& S S_{1}: \dot{x}(t)=A x(t)+B K_{1} x\left(t-\tau_{1}(t)\right), t \in\left[t_{0}, t_{1}\right] ; \\
& S U_{1}: \dot{x}(t)=A x(t)+B K_{1} x\left(t-\tau_{2}(t)\right), t \in\left[t_{1}, t_{2}\right] ; \\
& S S_{2}: \dot{x}(t)=A x(t)+B K_{2} x\left(t-\tau_{2}(t)\right), t \in\left[t_{2}, t_{3}\right] ; \\
& S U_{2}: \dot{x}(t)=A x(t)+B K_{2} x\left(t-\tau_{1}(t)\right), t \in\left[t_{3}, t_{4}\right] ;
\end{aligned}
$$

\section{B. Global stability: minimum dwell time}

The minimum dwell time for $S S_{1}$ and $S S_{2}$ has to be calculated to guarantee the decreasing sequence of the values at the switching instants for each stable mode. Decreasing sequence in Fig. 1 implies that there exits a $\mu$, the smaller positive real such that:

$$
V_{i}\left(t, x_{t}, \dot{x}_{t}\right) \leq \mu V_{j}\left(t, x_{t}, \dot{x}_{t}\right), i, j \in\{1,2\} .
$$

This is implied by the following conditions:

$$
\begin{aligned}
& P_{i} \leq \mu P_{j}, \\
& e^{-2 \alpha_{i} h} S_{k i} \leq \mu e^{-2 \alpha_{j} h} S_{k j}, h \in\left[0, h_{k}\right], \\
& e^{-2 \alpha_{i} h} R_{k i} \leq \mu e^{-2 \alpha_{j} h} R_{k j}, h \in\left[0, h_{k}\right], \\
& k=0,1,2,
\end{aligned}
$$

Here we give the analysis for the minimum dwell time calculation of $S S_{2}$, the same method can be applied for the case of $S S_{1}$. 


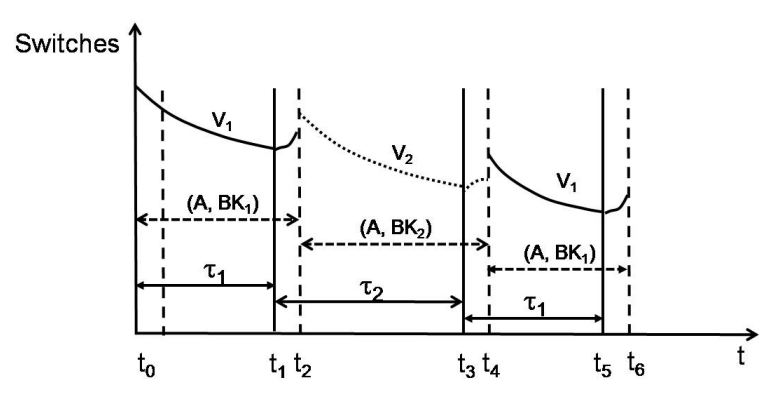

Fig. 1. Minimum dwell time

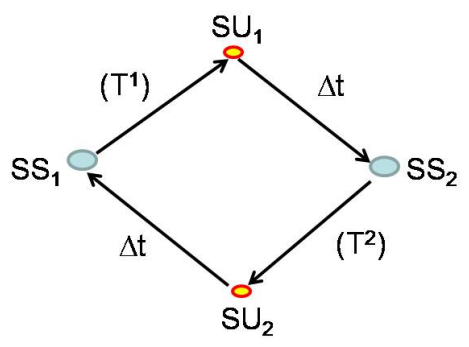

Fig. 2. Switch among four modes.

So the global stability problem can be solved by completing the following three small ones:

(1) Exponential stabilization for each mode: adapt Theorem 1, applying the bijective transformation to some matrices as showed in [10], the maximum values of $\alpha_{1}$ and $\alpha_{2}$, respectively representing the exponential decrease decay of each mode, can be obtained according to the similar LMIs conditions as (14) and (15) for $j=1$, (19) and (20) for $j=2$, where

$$
\begin{aligned}
& \tilde{R}_{i j}=e^{-2 \alpha h_{i+1}} R_{i j}, \tilde{S}_{i j}=e^{-2 \alpha h_{i+1}} S_{i j}, \hat{S}_{i j}=e^{-2 \alpha h_{i}} S_{i j}, \\
& \Phi_{11}^{j}=A P_{2 j}+P_{2 j}^{T} A^{T}+S_{0 j}-e^{-2 \alpha h_{1}} R_{0 j}+2 \alpha_{j} P_{j}, \\
& \Phi_{12}^{j}=P_{j}-P_{2 j}+\varepsilon P_{2 j}^{T} A,(\varepsilon>0), \\
& \Phi_{22}^{j}=-\varepsilon P_{2 j}-\varepsilon P_{2 j}^{T}+\sum_{i=0}^{2}\left(h_{i+1}-h_{i}\right)^{2} R_{i j}, \\
& \Phi_{16}^{j}=B M_{j}, \Phi_{26}^{j}=\varepsilon B M_{j}, \phi_{3}^{(1)}=-\left(\tilde{S}_{0 j}+\tilde{R}_{0 j}-\hat{S}_{1 j}\right), \\
& \phi_{4}^{(1)}=-\left(\tilde{S}_{1 j}+\tilde{R}_{2 j}-\hat{S}_{2 j}\right) \\
& \phi_{5}^{(1)}=-\left(h_{2}-h_{1}\right)\left(\tau-h_{1}\right) \tilde{R}_{1 j}, \\
& \phi_{6}^{(1)}=-\left(h_{2}-h_{1}\right)\left(h_{2}-\tau\right) \tilde{R}_{1 j}, \\
& \phi_{3}^{(2)}=-\left(\tilde{S}_{0 j}+\tilde{R}_{0 j}+\tilde{R}_{1 j}-\hat{S}_{1 j}\right), \\
& \phi_{4}^{(2)}=-\left(\tilde{S}_{1 j}+\tilde{R}_{1 j}-\hat{S}_{2 j}\right), \\
& \phi_{5}^{(2)}=-\left(h_{3}-h_{2}\right)\left(\tau-h_{2}\right) \tilde{R}_{2 j}, \\
& \phi_{6}^{(2)}=-\left(h_{3}-h_{2}\right)\left(h_{3}-\tau\right) \tilde{R}_{2 j} .
\end{aligned}
$$

Then, the two gains can be calculated as $K_{j}=M_{j} P_{2 j}^{-1}$.

(2) Calculation of the smaller positive $\mu$ according to the equation (24) and (25).

(3) Exponential decay for the mode $S U_{1}$ : Apply Theorem 1 for the mode $S U_{1}$ with time-delay $\tau_{2}(t) \in\left[h_{2}, h_{3}\right]$, with $K_{1}$ calculated from the step (1). Here, the timedelay of switching signal is supposed to be constant, then, the exponential value $\alpha_{1}^{\prime}$ can be calculated. If this mode is unstable, the value of $\alpha_{1}^{\prime}$ is negative, which will be taken into account in the calculation of the minimum dwell time for $S S_{2}$.

Notice that:

$$
\begin{aligned}
V_{2}\left(t_{2}, x_{t_{2}}, \dot{x}_{t_{2}}\right) & \leq \mu V_{1}\left(t_{2}, x_{t_{2}}, \dot{x}_{t_{2}}\right) \\
& \leq \mu e^{-2 \alpha_{1}^{\prime}\left(t_{2}-t_{1}\right)} V_{1}\left(t_{1}, x_{t_{1}}, \dot{x}_{t_{1}}\right) \\
& \leq \mu e^{-2 \alpha_{1}^{\prime}\left(t_{2}-t_{1}\right)-2 \alpha_{1}\left(t_{1}-t_{0}\right)} V_{1}\left(t_{0}, x_{t_{0}}, \dot{x}_{t_{0}}\right)
\end{aligned}
$$

$\mu e^{-2 \alpha_{1}^{\prime}\left(t_{2}-t_{1}\right)-2 \alpha_{1}\left(t_{1}-t_{0}\right)} \leq 1$ is sufficient to prove the switching stability from $S S_{1}$ to $S S_{2}$ showed in Fig. 23, so the minimum dwell time for $S S_{1}$ is:

$$
t_{1}-t_{0}=\frac{\ln (\mu)-2 \alpha_{1}^{\prime}\left(t_{2}-t_{1}\right)}{2 \alpha_{1}} .
$$

The minimum dwell time for $S S_{2}$ can be calculated in the same way and the value is:

$$
t_{3}-t_{2}=\frac{\ln (\mu)-2 \alpha_{2}^{\prime}\left(t_{4}-t_{3}\right)}{2 \alpha_{2}} .
$$

\section{EXAMPLE}

The model of a linear system is showed in equation (30).

$$
\dot{x}(t)=\left[\begin{array}{cc}
0 & 1 \\
0 & -10
\end{array}\right] x(t)+\left[\begin{array}{c}
0 \\
0.024
\end{array}\right] u(t-\tau(t)) .
$$

Consider a linear state feedback controller, i.e., no switch is concerned. Applying the same LKF as equation (3) with $S_{2}=0, R_{2}=0$ and $\tau(t) \in[0.01,0.4]$, the maximum exponential decay value of $\alpha$ is obtained as 0.88 . While, considering two intervals of delay with [0.01, 0.05[ and [0.05, 0.4], we can get better performance. It means that the control gain switches when the delay crosses the value of $0.05 \mathrm{sec}$. According to the approach in this paper, the decay rate ensuring the exponential stability in every modes are: $\alpha_{1}=1.1$, $\alpha_{2}=0.89$ and the maximum exponential values for $S U_{1}$ and $S U_{2}$ respectively are: $\alpha_{1}^{\prime}=-2.35, \alpha_{2}^{\prime}=1.1$. That is to say, the mode $S U_{1}$ is unstable.

The gains $K_{i}(i=1,2)$ and $\mu$ are:

$$
\left[\begin{array}{l}
K_{1} \\
K_{2}
\end{array}\right]=\left[\begin{array}{ccc}
-1639 & -164 \\
-742 & -74
\end{array}\right], \mu=1.4 .
$$

Considering the delay of the switching signal is $0.1 \mathrm{sec}$, so the minimum dwell time for staying in the mode $S S_{1}$ before switching to the mode $S S_{2}$ can be achieved as $0.35 \mathrm{sec}$ and the one for stay in the mode of $S S_{2}$ is $0.06 \mathrm{sec}$. In Fig. 3, when the condition of minimum dwell time is satisfied, the global stability is guaranteed.

As we can find in the equations (28) and (29), the values of dwell time depend on the value of the exponential decay, so we have to make a tradeoff between 


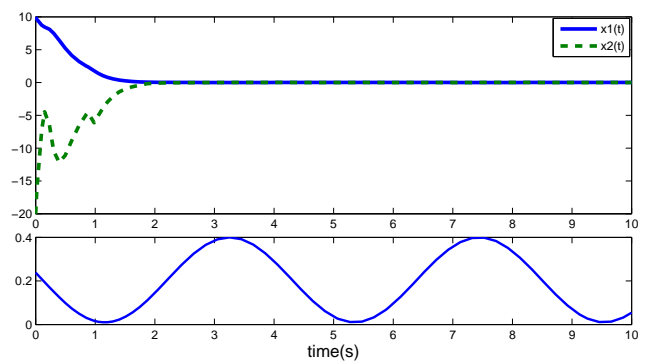

Fig. 3. Solution of (30) for $\tau(t)=0.19 \sin (1.5 t)+0.21$.

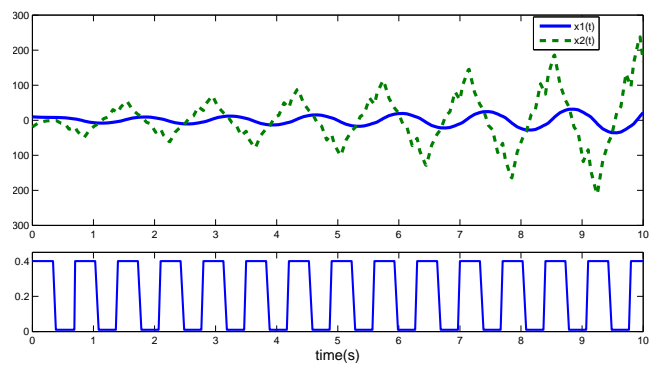

Fig. 4. Solution of (30) for $\tau(t)$, a pulse signal.

the bigger value of $\alpha$ and the smaller dwell time. For example, we can get $\alpha_{1}=3.8$ but with the dwell time for $S S_{2}$ equals $1.15 \mathrm{sec}$. Fig. 4 shows that when the dwell time in the mode $S S_{2}$ is not sufficient, the system may become unstable.

These new conditions can be also used to enlarge the interval of admissible delays. For example, considering a linear state feedback controller i.e. without QoS adaptation, the linear model (30) can be stabilized for delays in $[0.01,1.26]$ (largest interval possible). By considering the delay intervals $[0.01,1.2]$ and $[1.2,2]$ one can find a switching controller with a decay rate of $\alpha=0.1$ and a minimum dwell time of 3.38 .

\section{CONCLUSION}

In this paper, the stabilization was mainly focused on the switched time-delay system with the delayed switching signal. Exponential stability is obtained for the principle switching modes, and global stability is considered by taking into account the period when the switching signal has not yet been detected. The result can be adapted to verify the stability of more general switched time-delay systems with temporary uncertain switching signals. In addition, it can be also applied to packet loss problems in the case of networked control systems, where the loss of packets cannot be detected immediately by the system.

\section{REFERENCES}

[1] Chen C.-C., Hirche S., and Buss M., "Towards quality-of-service control of networked control systems: a switched time delay systems approach," Proceedings of the 2006 IEEE International Conference on Control Applications, October 2006.

[2] Chiasson J. and Loiseau J.J., Applications of time delay systems. Springer, 2007, vol. 352.

[3] Fridman E., "New Lyapunov-Krasovskii functionals for stability of linear retarded and neutral type systems," Systems \& Control Letters, vol. 43, pp. 309-319, 2001.

[4] Gu K., Kharitonov V., and Chen J., "Stability of time-delay systems," Birkhauser: Boston, 2003.

[5] He Y., Wang Q-G., Lin C., and Wu M., "Delay-range-dependent stability for systems with time-varying delay," Automatica, vol. 43, no. 2, pp. 371-376, 2007.

[6] He Y., Wu M., She J.H., and Liu G.P., "Parameter-dependent lyapunov functional for stability of time-delay systems with polytopic-type uncertainties," IEEE Transactions on Automatic Control, vol. 49, pp. 828-832, 2004.

[7] Hespanha J.P. and Morse A.S., "stability of switched systems with average dwell-time," Proceedings of the $38^{\text {th }}$ Conference on Decision \& Control, pp. 2655-2660, December 1999.

[8] Hetel L., Daafouz J., and Iung C., "Stability analysis for discrete time switched systems with temporary uncertain switching signal," 2007 the $46^{\text {th }}$ IEEE Conference on Decision \& Control, pp. 5623-5628, Decembre 2007.

[9] Hirche S., Chen C.-C, and Buss M, "Performance oriented control over networks -switching controllers and switched time delay-," Proceedings of the 45th IEEE Conference on Decision \& Control, December 2006.

[10] Jiang W.-J., Kruszewski A., Richard J.-P., and Toguyeni A., "A gain scheduling strategy for the control and estimation of a remote robot via internet," The 27th Chinese Control Conference, July 2008.

[11] Liberzon D., Switching in Systems and Control, T. Basar, Ed. Birkhäuser, 2003.

[12] Liberzon D., Hespanha J.P., and Morse A.S., "Stability of switched systems: a lie-algebraic condition," Systems Control Lett., vol. 37, pp. 117-122, 1999.

[13] Narendra K.S. and Balakrishnan J., "A common lyapunov function for stable lti systems with commuting a-matrices," IEEE Trans. Automat. Control, vol. 39, pp. 2469-2471, 1994.

[14] Niculescu S.-I., Delay effects on stability: a robust control approach. Springer, 2001, vol. 269.

[15] Park P.G. and Ko. J.W., "Stability and robust stability for systems with a time-varying delay," Automatica, vol. 43, pp. 1855-1858, 2007.

[16] Richard J.-P., "Time delay systems: an overview of some recent advances and open problems," Automatica, vol. 39, pp. 16671694, 2003.

[17] Sun X.-M., Zhao J., and Hill D., "Stability and $l_{2}$-gain analysis for switched delay systems," Automatica, vol. 42, pp. 1769-1774, 2006.

[18] Sun Y.G. and Wang L., "Stability of switched systems with timevarying delays: delay-dependent common lyapunov functional approach," Proceedings of the 2006 American Control Conference, June 2006.

[19] Xie G. and Wang L., "Stabilization of switched linear systems with time-delay in detection of switching signal," Mathematical analysis and application, vol. 305, pp. 277-290, 2005.

[20] Yan P. and Özbay H., "Stability analysis of switched time delay systems," SIAM Journal on Control and Optimization, vol. 47, no. 2, pp. 936-949, 2008. 